\title{
Phosphodiesterase type 5 inhibitor therapy provides sustained relief of symptoms among patients with chronic pelvic pain syndrome
}

\author{
Kevin Pineault, Shagnik Ray, Andrew Gabrielson, Amin S. Herati \\ Department of Urology, The James Buchanan Brady Urological Institute, Johns Hopkins University School of Medicine, Baltimore, MD, USA \\ Contributions: (I) Conception and design: K Pineault, AS Herati; (II) Administrative support: AS Herati; (III) Provision of study materials or patients: \\ K Pineault, AS Herati; (IV) Collection and assembly of data: All authors; (V) Data analysis and interpretation: K Pineault, S Ray; (VI) Manuscript \\ writing: all authors; (VII) Final approval of manuscript: All authors. \\ Correspondence to: Amin S. Herati, MD. Department of Urology, The James Buchanan Brady Urological Institute, Johns Hopkins University School \\ of Medicine, Park Building 217, 600 North Wolfe St., Baltimore, MD 21287, USA. Email: aherati1@jhmi.edu.
}

\begin{abstract}
Background: Chronic prostatitis/chronic pelvic pain syndrome type III (CP/CPPS) is associated with pelvic pain, sexual dysfunction and irritative voiding. Sustained symptom relief has proven difficult with alpha blockers, antibiotics, and nonsteroidal anti-inflammatory drugs (NSAIDs). Phosphodiesterase type 5 (PDE5) inhibitors (PDE5is) have the potential to alleviate bladder urgency, relax the pelvic floor, and correct underlying erectile dysfunction; however, few studies have investigated the application of PDE5i's to CP/ CPPS. The purpose of this study was to assess the effect of long-term PDE5i therapy on symptoms among patients with diagnosed CP/CPPS.
\end{abstract}

Methods: A group of patients older than 18 years diagnosed with CP/CPPS presenting from 2009 to 2018 were followed prospectively while they were being prescribed off-label PDE5i therapy for symptoms. National Institute of Health chronic prostatitis symptom index (CPSI) scores before PDE5i therapy initiation and after at least 3 months were utilized to assess impact on symptoms.

Results: A total of 25 patients (mean age $44.4 \pm 12.9$ years) met study criteria. The mean duration of PDE5i therapy was $1.3 \pm 1.6$ years. Continued use of daily PDE5is was associated with significant decreases in total CPSI, pain, urinary symptom and quality of life scores [total CPSI: -12.8 , standard deviation (SD) 9.5; pain: -6.1 , SD 4.1; urinary symptoms: -2.4 , SD 2.1; quality of life: -4.5 , SD 3.9; $\mathrm{P}<0.001$ ].

Conclusions: This prospective data suggests that PDE5i therapy is associated with durable decreases in CP/CPPS symptoms past 3 months.

Keywords: Prostatitis; phosphodiesterase inhibitors; quality of life (QoL)

Submitted Oct 09, 2019. Accepted for publication Feb 14, 2020.

doi: $10.21037 /$ tau.2020.03.05

View this article at: http://dx.doi.org/10.21037/tau.2020.03.05

\section{Introduction}

Chronic prostatitis/chronic pelvic pain syndrome type III (CP/CPPS) is a chronic pain disorder associated with sexual dysfunction and irritative voiding symptoms (1). Effective, long-lasting CP/CPPS therapy has proven difficult with alpha blockers, antibiotics, and nonsteroidal antiinflammatory drugs (NSAIDs) in addition to pelvic floor therapy (PFT), stress management, prostatic massage, nerve stimulation and behavioral therapy (2-8). Even with these available therapies, patients typically suffer a fluctuating chronic course of urinary symptoms, pain, and ejaculatory dysfunction $(1,9)$. Due to poor long-term treatment success, CP/CPPS can impact quality of life (QoL) even more than congestive heart disease and diabetes mellitus and can lead to decreased overall function compared to the general male population (10). 
Despite the lack of adequate long-term symptom management of CP/CPPS, there has been little investigation into the potential of phosphodiesterase type 5 (PDE5) inhibitors. PDE5 inhibitors (PDE5is) improve lower urinary tract symptoms (LUTS) in men with benign prostatic hyperplasia (BPH), pulmonary arterial hypertension $(\mathrm{PAH})$, complex regional pain syndrome and erectile dysfunction (ED) by relaxing the smooth muscle fibers of the prostate through modulation of nitric oxide (NO)-cyclic guanosine monophosphate (cGMP) signaling (3,11-15). Recent investigation showed promising reductions in symptoms and QoL using the NIH Chronic Prostatitis Symptom Index (CPSI) and International Prostate Symptoms Score (IPSS) among patients with CP/ CPPS after 3 months of therapy $(16,17)$.

In this study, we prospectively assessed symptoms and QoL among patients who had been diagnosed with CP/ CPPS at the Brady Urological Institute while taking a daily PDE5i. We hypothesized that long-term PDE5i use would lead to lasting reduction of symptoms over time and improved QoL. Therefore, the aims of this study were to determine (I) CPSI before PDE5i use, (II) CPSI after at least 3 months of use, and thus (III) resulting changes in QoL among patients with CP/CPPS.

\section{Methods}

\section{Study design}

After institutional review board (IRB) approval and patient consent were obtained, on all patients with CP/CPPS who were prescribed a PDE5i, specifically Tadalafil, as off-label therapy at the Brady Urological Institute were followed prospectively for at least 3 months follow-up between 2009 and 2018. Patients were selected based on inclusion/ exclusion criteria including (I) confirmed diagnosis of CP/ CPPS with symptoms for at least 3 months, (II) age $\geq$ 18 years old, (III) no other urological conditions or urinary tract infections present, and (IV) no current use of alpha blockers, NSAIDs, or antibiotics although failed use of these medications or PFT did not exclude patients. Patients who did not follow up or continue consistent daily PDE5i therapy were also excluded. These patients were identified by a response of "No" to the question, "Did you take 5 $\mathrm{mg}$ tadalafil daily as prescribed since therapy initiation?" Patients were also excluded if other medications were started for CP/CPPS or another co-existing medical condition between start of PDE5i therapy and the follow up visit. Of note, three patients included had a diagnosis of ED.

\section{NIH CPSI and outcome variables}

The NIH CPSI questionnaire was utilized to determine total CPSI score, urinary symptoms, pain, and QoL outcome measures (17). The NIH CPSI and demographic information including age, gender, and past medical history was gathered using clinic notes and discharge summaries. The NIH CPSI scores specifically recorded were CPSI scores documented before patients were started on PDE5i treatment at an initial visit and scores assessed at least 3 months after continuing therapy.

\section{Statistical analysis}

In addition to descriptive statistical results to describe patient characteristics and pre- and post-PDE5i CPSI scores, paired $t$-tests were used to evaluate the outcome variable of total CPSI, urinary symptom, pain, and QoL scores. Logistic regression modeling was used to evaluate the association between time duration between CP/CPPS diagnosis and initiation of PDE5i therapy, duration of PDE5i therapy, and age on changes in total CPSI, urinary symptom, pain, and QoL scores before and after PDE5 therapy. Statistical significance was considered if $\mathrm{P}$ value $<0.05$. Statistical analyses were performed using STATA v.15.0 (STATA Corp., College Station, TX, USA, 2017).

\section{Results}

Twenty-five patients presenting to the Brady Urological Institute between 2009 and 2018 who satisfied study criteria were enrolled in this study (Table 1). The patients had a mean age of $44.4 \pm 12.9$ years. All patients had a diagnosis of $\mathrm{CP} / \mathrm{CPPS}$ although three patients also had a diagnosis of ED (12\%). The mean duration from diagnosis of CP/CPPS to initiation of PDE5i therapy was $5.8 \pm 5.2$ years. The mean duration of PDE5i treatment in this population of patients was $1.3 \pm 1.6$ years. Table 1 displays the specific changes in pain, urinary, QoL, and total CPSI scores for each patient at the follow up visit after PDE5i use, and Figure 1 displays mean changes in each score for the entire study population. Total CPSI scores were found to significantly decrease after at least 3 months of PDE5i therapy [mean change of -12.9 , standard deviation (SD) $9.6, \mathrm{P}<0.001$ ]; however, interestingly two patients demonstrated no change in total 
Table 1 Patient demographics, prior therapies, and responses to PDE inhibitor therapy

\begin{tabular}{|c|c|c|c|c|c|c|c|c|}
\hline Patient ID & $\begin{array}{c}\text { Age } \\
\text { (years) }\end{array}$ & $\begin{array}{c}\text { Failed prior } \\
\text { treatment (yes }=1 \text { ) }\end{array}$ & $\begin{array}{c}\text { Current PFT use } \\
\quad(\text { yes }=1)\end{array}$ & $\begin{array}{c}\text { Duration of } \\
\text { therapy (years) }\end{array}$ & $\begin{array}{l}\text { Change } \\
\text { in pain }\end{array}$ & $\begin{array}{l}\text { Change in } \\
\text { urinary }\end{array}$ & $\begin{array}{l}\text { Change } \\
\text { in QoL }\end{array}$ & $\begin{array}{c}\text { Change in total } \\
\text { CPSI }\end{array}$ \\
\hline 1 & 47 & 0 & 0 & 0.75 & -5 & 0 & -3 & -8 \\
\hline 3 & 55 & 1 & 0 & 0.25 & -8 & -7 & -8 & -23 \\
\hline 4 & 60 & 1 & 0 & 0.99 & -5 & 4 & 0 & -1 \\
\hline 6 & 55 & 1 & 0 & 0.25 & -6 & -7 & -8 & -21 \\
\hline 7 & 31 & 1 & 0 & 0.83 & -11 & -8 & -10 & -29 \\
\hline 8 & 53 & 1 & 0 & 0.44 & -6 & -5 & -3 & -14 \\
\hline 9 & 47 & 0 & 1 & 0.99 & -4 & -2 & -7 & -13 \\
\hline 12 & 37 & 1 & 1 & 0.38 & -3 & -4 & -1 & -8 \\
\hline 13 & 46 & 0 & 0 & 0.86 & -4 & -2 & -3 & -9 \\
\hline 14 & 23 & 1 & 1 & 1.18 & -6 & -3 & -8 & -10.5 \\
\hline 15 & 32 & 0 & 1 & 0.30 & -5 & 0 & -1 & -6 \\
\hline 16 & 26 & 1 & 0 & 0.28 & -1 & -5 & -1 & -7 \\
\hline 17 & 66 & 1 & 1 & 0.25 & -10 & -1 & -4.5 & -15.5 \\
\hline 18 & 18 & 1 & 0 & 0.25 & -12 & -5 & -11 & -28 \\
\hline 19 & 46 & 0 & 0 & 0.30 & -9 & -1 & -3 & -13 \\
\hline Mean & $44.4 \pm 12.9$ & - & - & $1.3 \pm 1.6$ & $-6.1 \pm 4.1$ & $-2.4 \pm 3.0$ & $-4.6 \pm 4.0$ & $-12.9 \pm 9.6$ \\
\hline
\end{tabular}

PDE, phosphodiesterase; PFT, pelvic floor therapy; QoL, quality of life; CPSI, chronic prostatitis symptom index.

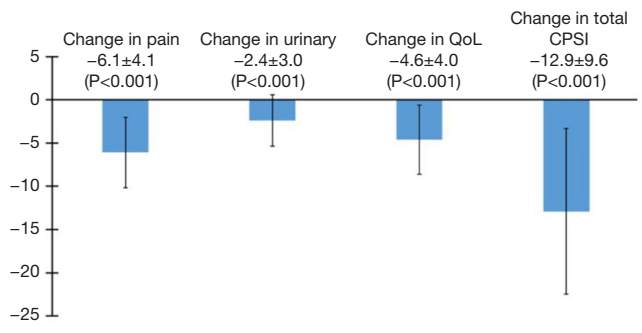

Figure 1 Differences in CPSI scores after at least 3 months of PDE5 inhibitor therapy for entire study population ( $n=25)$. QoL, quality of life; CPSI, chronic prostatitis symptom index; PDE5, phosphodiesterase type 5 .
CPSI score after use. Pain, urinary symptom, and QoL scores determined from the same NIH CPSI questionnaire also significantly decreased respectively (mean change in pain: $-6.1, \mathrm{SD} 4.1$; in urinary symptoms: -2.4 , SD 3.0; in QoL: -4.6, SD 4.0; $\mathrm{P}<0.001)$. Figure 2 further displays each patient's change in total CPSI, pain, urinary symptom and QoL scores with duration of PDE5i therapy to allow comparison to other patients in the study population with varying durations of therapy. Additional logistic regression analyses did not reveal any significant associations between duration between time of CP/CPPS diagnosis and PDE5i 

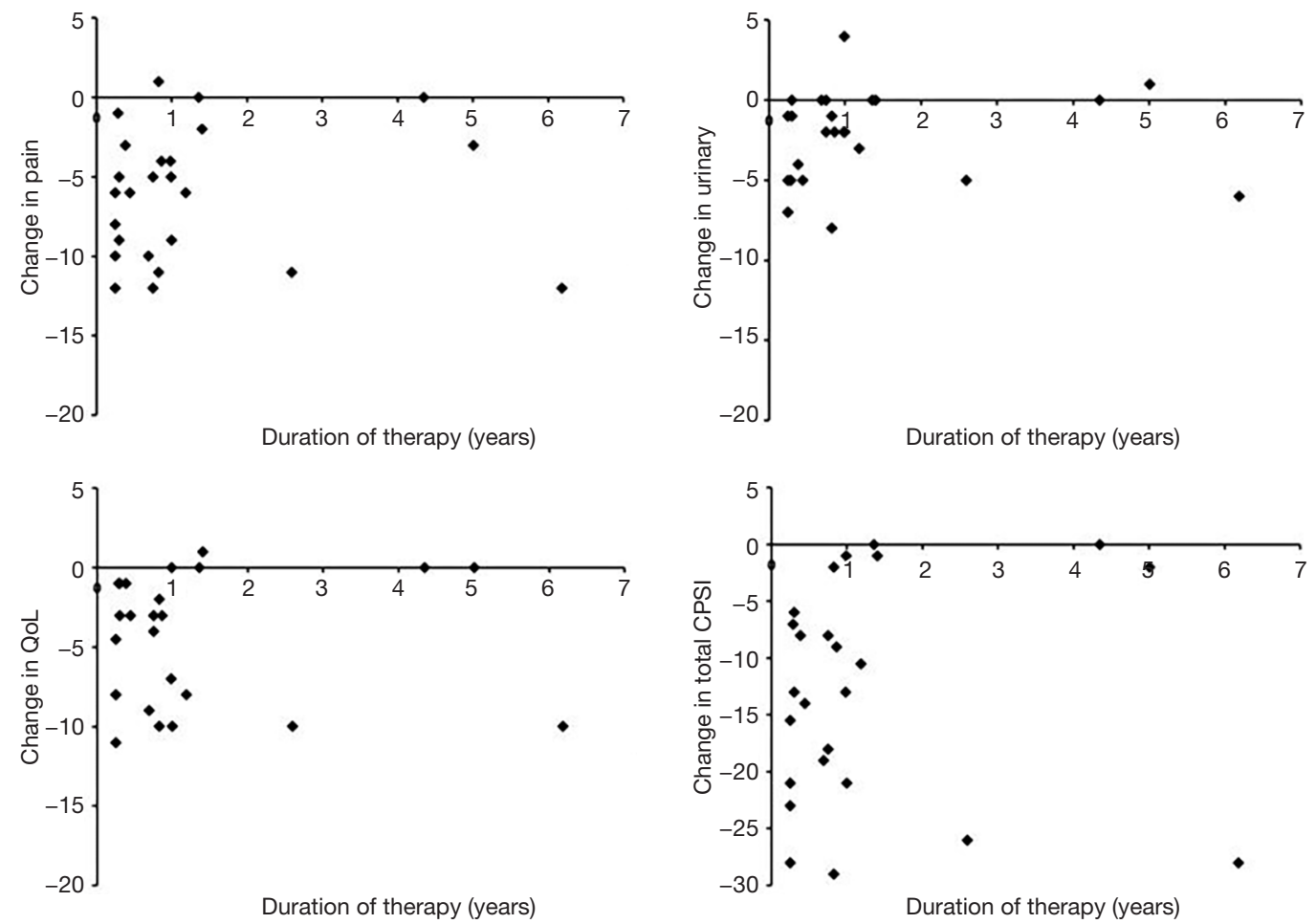

Figure 2 Change in pain, urinary, QoL, and total CPSI for specific PDE5 inhibitor therapy durations for each individual study patient. QoL, quality of life; CPSI, chronic prostatitis symptom index; PDE5, phosphodiesterase type 5.

initiation, duration of PDE5i therapy, and age, respectively, on changes in total CPSI score, urinary symptom score, pain score, and QoL scores before and after PDE5i therapy (data not included).

\section{Discussion}

The purpose of this study was to assess the effect of longterm (minimum of 3 months or longer) daily PDE5i therapy on symptoms and QoL among patients with diagnosed CP/ CPPS. We hypothesized that extended daily PDE5i therapy would result in significant decreases in symptoms and improvements in QoL for these patients. Current therapy options with alpha blockers, antibiotics and NSAIDs have not provided adequate long-term improvements among patients with CP/CPPS. To our knowledge, this is one of the only studies assessing the effects of PDE5i treatment for CP/CPPS past 3 months.

Our findings regarding improvements in CP/CPPS symptoms and QoL are consistent with the most recent literature. Among patients in our study, significant improvements all domains of the NIH CPSI questionnaire, including total score, urinary symptoms, pain, and QoL, were observed after long-term use of a PDE5i. A recent study of PDE5i use in men with CP/CPPS by Benelli et al. observed similar improvements in symptoms during oncedaily PDE5i treatment over the course of 3 months both on the NIH CPSI and IPSS among a similar population of men with CP/CPPS (16). Moreover, Sairam et al. was one of the first studies that found improvements in IPSS and QoL scores among men taking a PDE5i (e.g., Sildenafil) with co-existing LUTS (18). Other recent prospective studies in patients with BPH and CP/CPPS have further demonstrated significant improvements in LUTS with the CPSI and IPSS scoring tools and urodynamic parameters such as maximum flow rate (Qmax) $(19,20)$. Unfortunately, in the recent years, about $20 \%$ of all patients presenting with LUTS eventually need surgery for symptom relief (21). However, PDE5i treatment shows potential for addressing chronic symptoms of other disorders of the human urogenital tract in addition to CP/CPPS (22).

The mechanism behind development and recurrence of CP/CPPS symptoms with conventional treatment is complex and multifactorial. Dysregulated inflammation 
with elevated pro-inflammatory cytokines, androgen receptor dysfunction or low testosterone, and neurogenic inflammation lead to a state of systemic inflammation with decreased prostatic NO levels and increased Rho-Rho kinase activity, all of which may increase intraprostatic pressures $(1,23-26)$. Elevated interstitial pressures from decreased $\mathrm{NO}$ and increased Rho-Rho kinase activity have been shown to result in tissue ischemia and increased sensitivity within prostates as well $(23,27)$. By increasing intracellular cGMP, PDE5is induce smooth muscle relaxation within the prostate, and therefore, improve CP/CPPS-related pain by reducing intraprostatic pressure $(17,26,28)$. Prior work with experimental autoimmune prostatitis models in rats demonstrated treatment with a PDE5i (e.g., Tadalafil) led to reduced tactile allodynia and inflammation (29). Tadalafil, compared to other PDE5is like Sildenafil and Vardenafil, has a 5-fold higher sensitivity for PDE11A, which is highly expressed in the human prostate (30). As a result of smooth muscle relaxation and reduced intraprostatic pressures, retrograde flow of urine in the transitional and peripheral zones of the prostate is reduced, decreasing recurrent episodes of prostatitis (31).

Our study has several limitations that merit discussion. Due to this study's prospective design at an academic urology department in a large urban setting with all patient's seen by the same provider (i.e., study principal investigator), there is a possibility of selection bias and limited external validity of our results to the general population of patients suffering from CP/CPPS. Twelve patients were excluded from the final analysis because of incomplete follow-up or missing information on patient charts, which introduces potential bias in not accounting for non-responders to therapy. We note that two patients had zero change in total CPSI scores (Table 1, Figure 2), therefore, missing these 12 patients and potential non-responders limits the impact we can infer from this data. Importantly, there is a possibility of inconsistent adherence to daily PDE5i therapy due to various problems with accessing the medication, co-existing illness making patients avoid use intermittently, and forgetting to take the medication; therefore, potentially distorting the true effect and potential of PDE5is on reducing CP/CPPS symptoms. Duration of PDE5i therapy and subsequent follow up at minimum of 3 months was employed; however, mean duration of $1.3 \pm$ 1.6 years exhibits large varying follow up times. Therefore, we cannot confidently conclude PDE5i efficacy for CP/ CPPS. Although logistic regression analyses did not reveal any significant associations between time duration between
CP/CPPS diagnosis and initiation of PDE5i therapy, duration of PDE5i therapy, and age on CPSI scores, we believe this is likely due to the small number of patients included leading to under-powered analyses, which should be addressed in future longitudinal work with larger numbers of patients. Compared to the one other study examining PDE5i therapy with CP/CPPS, uroflowmetry parameters such as post-void residual urine (PVR) and Qmax and prostate size and prostate blood flow were not accessible for most patients and therefore excluded from analyses, limiting our ability to characterize functional changes in addition to symptomatic and QoL measures. Furthermore, although patients with concurrent use of standard therapies for CP/CPPS were excluded, patients' concurrent use of therapies such as alpha-blockers, antibiotics, and NSAIDs were not well documented. This information is critical when assessing a new drug's effectiveness prospectively in its off-label use and represents a limitation to this study. Moreover, we recognize there are other symptom scoring tools for LUTS (e.g., IPSS) and erectile function (e.g., International Index of Erectile Function) that were not employed regularly among patients on PDE5i therapy, and therefore, we recognize symptoms could have been more fully characterized in this population after treatment. Further longitudinal studies and possible randomized control trials comparing effects of placebo versus standard alpha blockers, antibiotics, and NSAIDs versus PDE5is will provide valuable insight into the potential of PDE5i therapy for CP/CPPS and are necessary to establish efficacy and safety compared to placebo and current treatment.

\section{Conclusions}

These data suggest that PDE5i therapy may be associated with sustained decreases in CP/CPPS symptoms after 1 year of follow-up. Current therapy options with alpha blockers, antibiotics and NSAIDs have not provided consistent longterm symptom improvements among patients with CP/ CPPS. Although this study examined a small population of these patients who completed a subjective CPSI symptom questionnaire, these results are promising, and we agree with colleagues that this therapy should be further explored for consideration.

\section{Acknowledgments}

Funding: None. 


\section{Footnote}

Conflicts of Interest: All authors have completed the ICMJE uniform disclosure form (available at http://dx.doi. org/10.21037/tau.2020.03.05). The authors have no conflicts of interest to declare.

Ethical Statement: The authors are accountable for all aspects of the work in ensuring that questions related to the accuracy or integrity of any part of the work are appropriately investigated and resolved. The study was approved by the Johns Hopkins IRB (No. IRB00199001) and written informed consent was obtained from all patients.

Open Access Statement: This is an Open Access article distributed in accordance with the Creative Commons Attribution-NonCommercial-NoDerivs 4.0 International License (CC BY-NC-ND 4.0), which permits the noncommercial replication and distribution of the article with the strict proviso that no changes or edits are made and the original work is properly cited (including links to both the formal publication through the relevant DOI and the license). See: https://creativecommons.org/licenses/by-ncnd/4.0/.

\section{References}

1. Pontari MA, Ruggieri MR. Mechanisms in prostatitis/ chronic pelvic pain syndrome. J Urol 2004;172:839-45.

2. Fink HA, Mac Donald R, Rutks IR, et al. Sildenafil for Male Erectile Dysfunction: A Systematic Review and Meta-analysis. Arch Intern Med 2002;162:1349-60.

3. Ahmed NS. Tadalafil: 15 years' journey in male erectile dysfunction and beyond. Drug Dev Res 2018. [Epub ahead of print].

4. Anothaisintawee T, Attia J, Nickel JC, et al. Management of chronic prostatitis/chronic pelvic pain syndrome. JAMA 2011;305:78-86

5. Magistro G, Wagenlehner FM, Grabe M, et al. Contemporary Management of Chronic Prostatitis/ Chronic Pelvic Pain Syndrome. Eur Urol 2016;69:286-97.

6. Cohen JM, Fagin AP, Hariton E, et al. Therapeutic intervention for chronic prostatitis/chronic pelvic pain syndrome (CP/CPPS): a systematic review and metaanalysis. PLoS One 2012;7:e41941.

7. Franco JV, Turk T, Jung JH, et al. Non-pharmacological interventions for treating chronic prostatitis/chronic pelvic pain syndrome. Cochrane Database Syst Rev 2018. Cochrane Database Syst Rev 2018;1:CD012551.

8. Pirola GM, Verdacchi T, Rosadi S, et al. Chronic prostatitis: current treatment options. Res Rep Urol 2019;11:165-74.

9. Murphy AB, Nadler RB. Pharmacotherapy strategies in chronic prostatitis/chronic pelvic pain syndrome management. Expert Opin Pharmacother 2010;11:1255-61.

10. McNaughton Collins M, Pontari MA, O'Leary MP, et al. Quality of Life Is Impaired in Men with Chronic Prostatitis: The Chronic Prostatitis Collaborative Research Network. J Gen Intern Med 2001;16:656-62.

11. Herati AS, Kohn TP, Butler PR, et al. Effects of Testosterone on Benign and Malignant Conditions of the Prostate. Curr Sex Health Rep 2017;9:65-73.

12. Gacci M, Andersson KE, Chapple C, et al. Latest Evidence on the Use of Phosphodiesterase Type 5 Inhibitors for the Treatment of Lower Urinary Tract Symptoms Secondary to Benign Prostatic Hyperplasia. Eur Urol 2016;70:124-33.

13. Chapple CR, Roehrborn CG, McVary K, et al. Effect of tadalafil on male lower urinary tract symptoms: an integrated analysis of storage and voiding international prostate symptom subscores from four randomised controlled trials. Eur Urol 2015;67:114-22.

14. Porst H, Kim ED, Casabe AR, et al. Efficacy and safety of tadalafil once daily in the treatment of men with lower urinary tract symptoms suggestive of benign prostatic hyperplasia: results of an international randomized, double-blind, placebo-controlled trial. Eur Urol 2011;60:1105-13.

15. Groeneweg G, Huygen FJ, Niehof SP, et al. Effect of tadalafil on blood flow, pain, and function in chronic cold complex regional pain syndrome: a randomized controlled trial. BMC Musculoskelet Disord 2008;9:143.

16. Benelli A, Mariani S, Varca V, et al. Once-daily $5 \mathrm{mg}$ tadalafil oral treatment for patients with chronic prostatitis/chronic pelvic pain syndrome. Ther Adv Urol 2018;10:377-81.

17. Litwin MS, McNaughton-Collins M, Fowler FJ, et al. The national institutes of health chronic prostatitis symptom index: development and validation of a new outcome measure. J Urol 1999;162:369-75.

18. Sairam K, Kulinskaya E, McNicholas TA, et al. Sildenafil influences lower urinary tract symptoms. BJU Int 2002;90:836-9.

19. Hiramatsu I, Tsujimura A, Soejima M, et al. Tadalafil is 
sufficiently effective for severe chronic prostatitis/chronic pelvic pain syndrome in patients with benign prostatic hyperplasia. Int J Urol 2020;27:53-7.

20. Oelke M, Giuliano F, Mirone V, et al. Monotherapy with Tadalafil or tamsulosin similarly improved lower urinary tract symptoms suggestive of benign prostatic hyperplasia in an international, randomised, parallel, placebocontrolled clinical trial. Eur Urol 2012;61:917-25.

21. Lu-Yao GL, Barry MJ, Chang CH, et al. Transurethral resection of the prostate among Medicare beneficiaries in the United States: time trends and outcomes. Prostate Patient Outcomes Research Team (PORT). Urology 1994;44:692-8; discussion 698-9.

22. Ückert S, Hedlund P, Andersson K, et al. Update on Phosphodiesterase (PDE) Isoenzymes as Pharmacologic Targets in Urology: Present and Future. Eur Urol 2006;50:1194-207.

23. Rees RW, Foxwell NA, Ralph DJ, et al. Y-27632, A RhoKinase Inhibitor, Inhibits Proliferation and Adrenergic Contraction of Prostatic Smooth Muscle Cells. J Urol 2003;170:2517-22.

24. Hayek OR, Shagsigh A, Kaplan SA, et al. Castration induces acute vasoconstriction of blood vessels in the rat prostate concomitant with a reduction of prostatic nitric

Cite this article as: Pineault K, Ray S, Gabrielson A, Herati AS. Phosphodiesterase type 5 inhibitor therapy provides sustained relief of symptoms among patients with chronic pelvic pain syndrome. Transl Androl Urol 2020;9(2):391-397. doi: 10.21037/ tau.2020.03.05 oxide synthase activity. J Urol 1999;162:1527-31.

25. Tsilidis KK, Rohrmann S, McGlynn KA, et al. Association between endogenous sex steroid hormones and inflammatory biomarkers in US men. Andrology 2013;1:919-28.

26. Burnett AL, Maguire MP, Chamness SL, et al. Characterization and localization of nitric oxide synthase in the human prostate. Urology 1995;45:435-9.

27. Mehik A, Hellstrom P, Nickel JC, et al. The chronic prostatitis-chronic pelvic pain syndrome can be characterized by prostatic tissue pressure measurements. J Urol 2002;167:137-40.

28. Takeda M, Tang R, Shapiro E, et al. Effects of nitric oxide on human and canine prostates. Urology 1995;45:440-6.

29. Okamoto K, Kurita M, Yamaguchi H, et al. Effect of tadalafil on chronic pelvic pain and prostatic inflammation in a rat model of experimental autoimmune prostatitis. Prostate 2018;78:707-13.

30. Fujishige K, Kotera J, Yuasa K, et al. The human phosphodiesterase PDE10A gene. Eur J Biochem 2000;267:5943-51.

31. Kirby RS, Lowe D, Bultitude MI, et al. Intra-prostatic Urinary Reflux: an Aetiological Factor in Abacterial Prostatitis. Br J Urol 1982;54:729-31. 